\title{
A Perspective on the Evolution of the International Trust Management Research Community in the Last Decade
}

\author{
Theo Dimitrakos \\ Security Futures Practice, BT Research \& Technology, UK \\ theo.dimitrakos@bt.com
}

\begin{abstract}
Year 2012 completes a decade since the iTrust research network was established in Europe. The international research community associated with iTrust is the predecessor of the IFIP Working Group 11.11 on Trust Management, the organization largely behind events such as the IFIPTM conference series. The completion of a decade since the establishment of iTrust also marks the first time that an international conference on Trust Management takes place in India, and indeed the first time that such a conference takes place outside of the aging "old world" economies of Europe, North America and Japan. This combination offers an excellent opportunity for a review of how we got here: the evolution of the international research community on trust management from 2002 to 2012. This review also offers a pre-text for surveying a selection of research results, research papers and innovative solution demonstrators that have been produce by the trust management community in the last decade.

This paper is the printed version of an invited keynote in IFIPTM 2012 conference that took place in Surat, India.
\end{abstract}

\section{$1 \quad$ Introduction}

The concepts of Trust and Trust Management in information systems and computer science attracted some attention in the late 1980's and the mid 1990's by pioneers who based themselves in sociological analyses such as Gambetta [1] or later in socioinspired information systems such as McKnight [2]. Soon after, the idea that it may be possible to treat trust as a computational concept has been put forward by Marsh in [3] while the idea that trust can be a mathematical framework to reason about aspects of trust in a social network appeared in the work of Jøsang in [4,5,6] and [7]. In parallel, the concept that by managing some symbolic representation of trust one can aid the automated verification of actions against security policies, was put forward by Blaze and his team in [8] and [9]. In this variant of access control, actions are allowed if sufficient credentials are presented, separating symbolic representation of trust from the actual person or its identity. Although in a different context and serving a different application, essentially the same concept that Blaze's team introduced in the late 1990's has found more recently a new home in the WS-Trust protocol [10] that underpins security token exchange in web services implementations. Blaze's concept is still how many researchers and practitioners in information security perceive "trust management" today, especially if they are unfamiliar with the wider, interdisciplinary body of research in trust and trust management. 
Reviewing the plethora of facets and definitions of "trust" and "trust management" is out of the scope of this short paper. Many informative surveys have written about trust and trust management definitions including an extensive one by Grandison [11] back in 2000, a shorter one by Ruohomaa in 2005 [12] and a more recent survey on reputation systems by Jøsang [13] in 2007. It is also out of scope to examine if the terms such as "trust" and "trust management" are meaningful without context and a pre-text or if indeed trust can be managed. My views on this have been already presented in [14] and in [16].

This paper and the associated keynote are about the formation and evolution of an international and interdisciplinary community in trust management. What triggered, in fact necessitated, the creation of this community was not only the results of the research mentioned above, but most notably a major event in the recent history of the developed world: a crisis often referred to as the (first) "dot com bubble burst" in 2000 - 2001. On Friday, March 10, 2000, the technology heavy NASDAQ Composite index, peaked at 5,048.62 (intra-day peak 5,132.52), more than double its value just a year before. The NASDAQ fell slightly after that, and continued until March 20, 2000, when the financial magazines shocked the market with cover stories reporting that, within a year, many highflying Internet upstarts will have used up all their cash and unless they scare up more cash, they will be facing a savage shakeout. For example an article in a highly reputable financial magazine reported a survey of the likely losers and highlighted that "America's 371 publicly traded Internet companies have grown to the point that they are collectively valued at $\$ 1.3$ trillion, which amounts to about $8 \%$ of the entire U.S. stock market"[15]. By 2001, a majority of the dot-com "miracle" companies ceased trading after burning through their venture capital, many having never made a "net" profit. The cause of the burst is often attributed to a combination of bad financial management and, most importantly, loss of consumer confidence as a result of unmet expectations for announced and oversold features that never materialized, negligent security and of poor customer experience.

\section{2 iTrust Working Group: Incubating of an International Trust Management Community in Europe}

The beginning of the first decade of the $21^{\text {st }}$ century was a time that governments, industry and academics in Europe and North America were concerned about an apparent loss of consumer confidence in on-line services. They came to realise that some stimulation was necessary in order to save the internet and on-line services economy, and to allow it to grow and excel again. During that period, I had moved on from Imperial College, London to work as a senior researcher for the Central Laboratory UK Research Councils; a research facilities laboratory that hosted the W3C Office for the UK and Ireland (in addition to some of the most advanced particle physics and space science facilities in Europe). At that time, I put forward the idea that trust in virtual communities and on-line services, and a framework to manage trust in these, is fundamental for re-building a strong on-line services economy [16,17]. 
The idea of research in trust and trust management in order to facilitate strengthening trust relationships in on-line communities and on-line service value networks was well received by the UK government, and found supporters among my colleagues in academia such as Professors Maibaum and Jones at King's College London, Professor Morris Sloman at Imperial College London, and Dr Simon Shiu in HP Labs Bristol.

At that time, I was already familiar with the work of Blaze's team, via the research of Grandison [18], then a $\mathrm{PhD}$ candidate in Sloman's group, as well as the relevance that Jøsang's ideas in automating trust-based decision making. I had also grown an interest in the interplay between trust and risk following joint work with Ketil Stolen on model-based security risk analysis, that was marked by the inception of the CORAS methodology in 2001 [24,25]. I was exposed to formal models and logicphilosophical investigations in trust via Maibaum [19], Jones [20], Falcone and Castelfranchi [21,22] as well as to legal analyses on trust via the work of a team researchers in law at the Norwegian Research Centre for Computer and Law (NRCCL). I was therefore convinced that a solution to the problem could not be simply technical (or mathematical) and that it could not ignore psychological, socio-technical, legal, operational and economic aspects.

At roughly the same period, other researchers in the Joint Research Centre of the European Commission were also concerned about the impact of trust in on-line services and markets [23]. Through my interactions with them and other European Commission officials, the concept of an international and truly multi-disciplinary network of researchers in trust and trust management for on-line community and online services was conceived, and the European Commission agreed to support such an initiative at least for an incubation period. The research network was to have a global reach from its birth, albeit a European core base, and it become truly global and selfsufficient once it matured. At that time colleagues alerted me that Professor Christos Nicolaou, then rector of the University of Crete, was also considering proposing a research network with a focus on computational trust in global computing infrastructures. After an initial discussion with Professor Nicolaou, it became clear to both of us that all these approaches and expertise to "trust" and "trust management" were complementary and should co-exist and co-evolve, cross-fertilize and eventually fuse in the same research community.

That was in essence the birth of the iTrust working group in 2001, the main predecessor of what is now the IFIP working group on Trust Management. The European Commission appreciated the interdisciplinary nature of the team that was attracted by the ambitious goals put forward, and agreed to support the creation of a research network in Trust Management from 2002 to 2005, under the short-name "iTrust" with the view that the community would have become global, self-funded and selfsufficient by 2005 .

\subsection{The Vision of an Early Working Group on Trust Management}

The vision of the iTrust working group was to bring together researchers and practitioners from a range of disciplines (computer science, sociology, economics, law, and philosophy) to develop models and techniques for dealing with trust in open dynamic 
systems. The group's aims were to explore the role of trust, and its interactions with security and authorization concerns for on-line virtual communities, value networks of on-line services and other dynamic open systems. We believed that effective trust modelling is an enabler for a range of new computing services including e-commerce, ubiquitous computing, grid computing, social networks and probably a variety of collaborative/cooperative online activities that we couldn't even imagine at that time.

For example, it was clear to us, at that time, that the sheer scale of the emerging global infrastructure, combined with the need for fully autonomous operation, surpass the usefulness of the advanced security infrastructures of that time including authorization services, public key infrastructures and certificate issuance and validation services. Possessing a certified identity in a dynamic and open environment does not a priori guarantee an acceptable behaviour and performance. In such systems, one cannot make informed decisions on access restrictions and controls, on selection of potential candidates to link in and interact with, or on what services to consume and how to consume them, on solely the basis of a certified identity. Mere knowledge of a certified identity alone is even less adequate for reasoning about the expected behaviour and dependability of entities for which no prior knowledge is available. Entities need to be distinguished not only based on their certified identities (which are static) but also based on their (un)expected, dynamically varying qualities that are relevant to the specific interaction context. Furthermore, such judgments, by necessity subjective due to the requirement for fully autonomous operation, need to be reviewed and possibly revised on a regular basis. For on-line services to achieve the same levels of acceptance as their conventional counterparts, trust management had to become an intrinsic part of on-line service provision.

Virtual community management, access management, business or social network partner selection, engagement in on-line transactions and e-commerce, and on-line service provision were some of the areas where we saw a needed for a practical, scalable and adaptable technology to capture, measure and manage the trusting relationships that underlie the interaction of on-line entities. Paving the way for such technology requires transfer of knowledge and close collaboration not only between academia and industry but also between different disciplines.

\subsection{First Steps of an International Research Community in Trust Management}

The iTrust research community soon expanded from its European base to include researchers from Australia, and North America. Members included legal experts, philosophers, psychologists, economists, and information / network security experts.

In 2003 the first collection of research results were published by the iTrust community in [26], following a conference in Crete, Greece. Research covered already a good mix of areas:

- Trust Models: including, for example, a model for "Regularity-Based Trust in Cyberspace" by Minsky, and a model for "Integrating Trustfulness and Decision Using Fuzzy Cognitive Maps" by Castelfranchi. 
- Policy-based systems: including, for example, a paper distilling the "Experience with the KeyNote Trust Management System: Applications and Future Directions" by Matt Balze and the KeyNote team, as well as a proposal of "Trust Management Tools for Internet Applications" by Grandison and Sloman.

- Credential disclosure negotiation: including an overview of trust negotiation principles and tools by Winslett in her paper "An Introduction to Trust Negotiation".

- Authentication systems and identity-based access control: including for example research on "Authenticated Dictionaries for Fresh Attribute Credentials" by William Winsborough and his team, and an "Implementation of an Agent-Oriented Trust Management Infrastructure Based on a Hybrid PKI Model" by Karabulut.

- Reputation systems: including for example work on "Simulating the Effect of Reputation Systems on E-markets" by Jøsang.

- Computational trust: including for example work on "Trusting Collaboration in Global Computing Systems" by Paddy Nixon and his team, as well as research on "Trust Propagation in Small Worlds" by Christian D. Jensen et al.

- Computer systems: including work on "Hardware Security Appliances for Trust" by Baldwin and Shiu, as well as work on "Managing Trust and Reputation in the XenoServer Open Platform" by Twigg et al.

- Early applications: including work "Towards the Intimate Trust Advisor" and a "Methodology to Bridge Different Domains of Trust in Mobile Communications" by Piotr Cofta et al.

- Socio-technical analyses: including for example an analysis on "Social Capital, Community Trust, and E-government Services" by Grimsley and Meehan, and a "A Trust Matrix Model for Electronic Commerce" by Yao-Hua Tan.

- Formal modelling of legal aspects: including for example an analysis on "Trust, Reliance, Good Faith, and the Law" by Giovanni Sartor et al.

The iTrust community continued to build on such works and produced more results that were published in [27] following a community event in Oxford, UK, in 2004. The event in Oxford emphasised on trust in large scale distributed systems and virtual organisations, on the use of recommendation and reputation systems in social networks and on-line services and included a mixture of technical results, application case studies and socio-technical and legal analyses.

- Large-scale systems and virtual organisations: included works such as "Engineering Trust Based Collaborations in a Global Computing Environment" by Terzis et al, "Towards Dynamic Security Perimeters for Virtual Collaborative Networks" by Djordjevic et al., "Trust, Security, and Contract Management Challenges for GridBased Application Service Provision" by Mac Randal et al., "Towards Trust Relationship Planning for Virtual Organizations" by Robinson, Haller et al., and "Deploying Trust Policies on the Semantic Web" by Matthews et al., and "W5: The Five W's of the World Wide Web" by Massimo Marchiori from the W3C.

- Recommendation and reputation systems: included, for example, "Using Trust in Recommender Systems: An Experimental Analysis" by Massa, et al, and "A Case for Evidence-Aware Distributed Reputation Systems: Overcoming the Limitations of Plausibility Considerations" by Philipp Obreiter. 
- Socio-technical analyses: included works such as "Human Experiments in Trust Dynamics" by C.M. Jonker, et al., "Modeling Controls for Dynamic Value Exchanges in Virtual Organizations" by Yao-Hua Tan et al., "Analyzing Correlation between Trust and User Similarity in Online Communities" by C.N. Ziegler, et al., "Managing Internet-Mediated Community Trust Relations" by Meehan et al., and "Trust Mediation in Knowledge Management and Sharing" by Castelfranchi.

- Legal analyses: included for example work on "Addressing the Data Problem: The Legal Framework Governing Forensics in an Online Environment" by Ian Walden.

The iTrust event in Oxford also fostered pioneering research investigating the interplay between on-line trust, risk and privacy as well as the role of trust in systems analysis and requirements engineering:

- Trust and risk: included for example "Analysing the Relationship between Risk and Trust" by Audun Jøsang and Stéphane Lo Presti, and "Using Risk Analysis to Assess User Trust: A Net-Bank Scenario" by Ketil Stølen.

- Trust and privacy: included for example "Trading Privacy for Trust" by Jean-Marc Seigneur, Christian Damsgaard Jensen, "Supporting Privacy in Decentralized Additive Reputation Systems" by Elan Pavlov et al.

- Trust in requirements engineering: included "Requirements Engineering Meets Trust Management: Model, Methodology, and Reasoning" by Fabio Massacci, John Mylopoulos et al.

The event in Oxford was also marked by an accompanying collection of tutorials and solution demonstrations that intensified knowledge transfer by bringing the iTrust community together with TrustCoM [28] - a major industry driven research project that brought together innovators from Atos, BT, BAe Systems, IBM, Microsoft and SAP with the aim to implement a collection standards-based web services technologies to facilitate secure and compliant business operation in virtual organisations.

Research in the iTrust community continued to produce strong results in 2005. The main community event of iTrust took place in Paris, France, with an emphasis on to computational trust, socio-technical and legal analyses [29]:

- Models of Computational trust: included works such as "Trust, Untrust, Distrust and Mistrust - An Exploration of the Dark(er) Side" by Marsh and Dibben, "A Representation Model of Trust Relationships with Delegation Extensions" by Lopez et al., "Towards a Generic Trust Model - Comparison of Various Trust Update Algorithms" by Kinateder et al., and "Towards an Evaluation Methodology for Computational Trust Systems" by J.-M. Seigneur et al.

- Socio-Technical analyses: included "Affect and Trust" by Lewis Hassel, "On Deciding to Trust" by Maria Fasli et al., and "Foraging for Trust: Exploring Rationality and the Stag Hunt Game" by Steven O. Kimbrough.

- Legal analyses: included works such as "Security and Trust in the Italian Legal Digital Signature Framework" by S. Zanero, and "Specifying Legal Risk Scenarios Using the CORAS Threat Modelling Language" by Mahler, Stølen et al. 
The iTrust event in Paris in 2005 included demonstrations of solution prototypes covering a wide range of security and trust applications. These included compliance enabling technology, trust assessment for grid computing and virtual organizations, trust and risk modeling tools, and requirements engineering environments. Short papers summarizing the innovative solutions being demonstrated were also published in [29].

Year 2006 was a decisive test for the trust management community as the iTrust working group had to prove its strength and maturity by continuing to operate as a self-funded research community without any formal subsidy or sponsorship by national governments or the European Union. That was when two colleagues from Italy, Fabio Martinelli and Fabio Massacci, offered to host an iTrust conference in Pisa, Italy supported by Ketil Stølen from Norway and William H. Winsborough from the USA. In recognition of the continuing quality of research produced within the iTrust community, Springer, who had been publishing the proceedings of all previous iTrust events, agreed to continue to publish. Results published in [30] included research in reputation systems, trust-based decision making, and socio-technical analyses:

- Recommendation and reputation systems: included for example "Generating Predictive Movie Recommendations from Trust in Social Networks" by Jennifer Golbeck, "PathTrust: A Trust-Based Reputation Service for Virtual Organization Formation" by Haller et al., and "Virtual Fingerprinting as a Foundation for Reputation in Open Systems" by A.J. Lee and M. Winslett.

- Trust-based decision making in trust networks: included for example "Exploring Different Types of Trust Propagation” by Jøsang and Marsh, "Gathering Experience in Trust-Based Interactions" by Terzis, "A Versatile Approach to Combining Trust Values for Making Binary Decisions” by Klos and Poutré, as well as "Provision of Trusted Identity Management Using Trust Credentials" by Pearson and Casassa Mont, and a "Bayesian Trust Framework for Pervasive Computing" by Quercia, Capra et al.

- Socio-technical analysis: included "Why We Need a Non-reductionist Approach to Trust" by Castelfranchi, "Modelling Trade and Trust Across Cultures" by C.M. Jonker et al., and "Being Trusted in a Social Network: Trust as Relational Capital" by Falcone et al.

Building on the tradition of previous events, iTrust 2005 included a collection of innovative solution demonstrations for a variety of applications including solutions for user classification, trust establishment, authorization services based on trust negotiation, and threat, vulnerability and risk assessment tools. Short papers summarizing these solutions were also published in [30].

\section{A Global Trust Management Community under IFIP}

At the side of iTrust 2006 event in Pisa, I met with Dr Fabio Martinelli, who was then leading the ERCIM European community on Trust and Security and with whom I was co-organizing a series of advanced, high-quality workshops on formal aspects of se- 
curity and trust $[31,32,33,34]$, and with Professor Javier Lopez, who was to become the first Chair of IFIPTM to exchange our views about facilitating a better future for the trust management research community after iTrust. We discussed ways for preserving the pace and securing the future growth of the trust management research community in Europe and methods to safeguard its progress towards self-sufficiency and globalization. We decided that, having succeeded the test of iTrust 2006, and given that the community members included a significant number of researchers based in north America and in Australasia, the time had come to formally recognize the global nature of the trust management community and propose the formation of a working group under the auspices of the International Federation of Information Processing (IFIP).

Beyond its good academic reputation, one of the reasons for looking at IFIP was its balanced and truly global reach including not only the established economies where iTrust was already strong but also many of the rapidly emerging internet economies in Asia, Middle East and Latin America, where we felt that on-line trust and trust management could be even more relevant in the future. At that critical time, Dr Steven Marsh contributed further to the establishment of an IFIP working group with a global reach, by facilitating a bridge between the iTrust community and a research community in North America who were running the PST event on Privacy, Security and Trust with the endorsement of the National Research Council of Canada.

In less than a year, IFIP agreed to form a working group on Trust Management under its technical committee on Security, while recognising and safeguarding the interdisciplinary nature of the trust management working group. In order to strengthen the bond between the iTrust and PST communities the first IFIP Trust Management conference took place in New Brunswick, Canada in 2007. The proceedings of that event were again published by Springer in [35], albeit under the IFIP (AICT) series instead of LNCS. The results in [35] included works on privacy, trust and legal analysis:

- Legal analysis: included "Pulling it all together...privacy, security, cybercrime and safety" by Parry Aftab.

- Trust models and trust management: included, for example, a paper presenting a "Private Distributed Scalar Product Protocol With Application To PrivacyPreserving Computation of Trust" by Danfeng Yao et al., research on "Trust Transfer in Distributed Systems" by Dulay et al., a "Content Trust Model for Detecting Web Spam" by Wang Wei and Zeng Guosun, and "A trust protocol for community collaboration" by S. Galice et al.

- Recommendation and Reputation systems: included "Exploiting Trust and Suspicion for Real-time Attack Recognition in Recommender Applications" by Bagheri and Ghorbani, research on "Self-Selection Bias in Reputation Systems" by M. Kramer and on "Resisting Sybils in Peer-to-peer Markets" by J. Traupman.

- Security and Privacy: such as "A Privacy-Aware Service Discovery Middleware for Pervasive Environments" by Issarny et al., and an "Analysis of the implicit trust within the OLSR protocol" by Adnane, et al., as well as "Negotiation for Authorisation in Virtual Organisations" by Paurobally and "A Geo Time Authentication System" by Mostarda, et al. 
In 2008, the IFIP working group on Trust Management had its annual event in Europe (Norway) where the focus was on trust modeling, recommendation and reputation systems, trust and privacy and socio-technical analyses [36]:

- Trust modeling: included "A Trust Evaluation Method Based on Logic and Probability Theory" by Reto Kohlas et al., "An Intensional Functional Model of Trust" by Kaiyu Wan, and A UML-based Method for the Development of Policies to Support Trust Management" by Ketil Stølen et al.

- Recommendation and reputation: included "Trust-Based Collaborative Filtering" and "SOFIA: Social Filtering for Robust Recommendations" by Licia Capra et al., "Continuous Ratings in Discrete Bayesian Reputation Systems" by Audun Jøsang et al., "Modeling Trust for Recommender Systems using Similarity Metrics" by Georgios Pitsilis, and "A Robust and Knot-Aware Trust-Based Reputation Model" by Nurit Gal-Oz, et al.

- Privacy and trust: included "A Model for Reasoning About the Privacy Impact of Composite Service Execution in Pervasive Computing" by Valérie Issarny, "Protecting Location Privacy through Semantics-aware Obfuscation Techniques" by Elisa Bertino et al., and an "Automatic Verification of Privacy Properties in the Applied pi Calculus" by Mark Ryan et al.

- Socio-technical analysis: included "Cooperation in Growing Communities" by Rowan Martin-Hughes and "The North Laine Shopping Guide: A Case Study in Modelling Trust in Applications" by Anirban Basu.

A collection of new technology demonstrations were also shown in IFIPTM 2008 including a stochastic reputation service for virtual organizations, a solution for monitoring application services, and a trust-based personalized travel guide. Short papers presenting these solutions were also included in [36].

As the trust management community had already developed critical masses in northern Europe and North America, the third Trust Management community took place in Purdue University, West Lafayette, Indiana, USA. Research reported in [37] focused on social aspects and usability, trust reasoning, trust and risk, privacy and data security, and recommendation and reputation systems:

- Social aspects and usability: including "Spiral of Hatred: Social Effects in BuyerSeller Cross-Comments Left on Internet Auctions" by Radoslaw Nielek, et al, and "Graphical Passwords as Browser Extension: Implementation and Usability Study" by Kemal Bicakci et al.

- Trust reasoning: including "Elimination of Subjectivity from Trust Recommendation" by Elisa Bertino, et al., and "Trust-Enhanced Recommender Systems for Efficient On-Line Collaboration" by Georgios Pitsilis, et al.

- Privacy and Data security: including "Security in Wiki-Style Authoring Systems" by Christian Damsgaard Jensen, "On Usage Control in Data Grids" by Fabio Martinelli et al., and "Detection and Prevention of Insider Threats in Database Driven Web Services" by Danfeng Yao et al. 
- Information sharing and trust negotiation: including "A Framework for Trustworthiness-Centric Information Sharing” by Ravi S. Sandhu et al., and "A Reconfigurable Framework for Trust Negotiation" by Marianne Winslett, et al.

- Recommendation and reputation systems: including "Comparison of the Beta and the Hidden Markov Models of Trust in Dynamic Environments" by Marie Elisabeth Gaup Moe, et al., and "Evaluating the STORE Reputation System in MultiAgent Simulations" by Yücel Karabulut et al., as well as "Employing Key Indicators to Provide a Dynamic Risk Picture with a Notion of Confidence" by Ketil Stølen et al.

Following a community meeting at IFIPTM 2009, a restructuring of the working group to its current form was implemented and that came together with a reaffirmation of the commitment of the trust management research community to pursue its goal of a truly global reach. Consequently, Professor Yuko Murayama offered to host a community event in Morioka, Iwate, Japan for 2010. This would be the first time that a trust management conference was held in Japan. The IFIPTM 2010 was the first conference in the series to take place in the Far East and, through its success, offered a unique opportunity for all relevant research communities in Japan to be exposed to, engage in, trust management research. Although the Japanese economy is very similar to those of Europe and North America, IFIPTM 2010 validated that trust management is also appealing to societies with a different structure and societal fabric than those of Europe and North America. Research results published in [38] included:

- Privacy and trust: including "Schemes for Privately Computing Trust and Reputation" by Nurit Gal-Oz et al., and "Self-service Privacy: User-Centric Privacy for Network-Centric Identity” by José M. del Álamo, et al.

- Trust Models: including "Non-monotonic Trust Management for Distributed Systems" by Naranker Dulay et al., and "Implementation and Performance Analysis of the Role-Based Trust Management System, RT ${ }^{\mathrm{C}}$ " by William Winsborough et al.

- Experimental and Experiential trust: including "Leveraging a Social Network of Trust for Promoting Honesty in E-Marketplaces" by Kate Larson et al., "Does Trust Matter for User Preferences? A Study on Epinions Ratings" by Georgios Pitsilis, et al., and 'Bringing the Virtual to the Farmers' Market: Designing for Trust in Pervasive Computing Systems" by Ian Wakeman, et al.

- Security and trust: including a "Visitor Access Control Scheme Utilizing Social Relationship in the Real World" by Gen Kitagata et al., and "Metric Strand Spaces for Locale Authentication Protocols" by Joshua D. Guttman et al., as well as "An Enterprise Service Bus for Access and Usage Control Policy Enforcement" by Gabriela Gheorghe, et al.

In 2011, IFIPTM returned to Europe and was hosted in Copenhagen, Denmark. The research published in [38] included works in trust models, reputation systems, social aspects and usability, and trust / privacy in the cloud: 
- Trust Modeling: such as "From Access Control to Trust Management, and Back A Petition" by Dieter Gollmann, and "Composing Trust Models towards Interoperable Trust Management" by Valérie Issarny, et al.

- Recommendation and reputation systems: such as "Detecting and Reacting to Changes in Reputation Flows" by Sini Ruohomaa et al., and "From Reputation Models and Systems to Reputation Ontologies" by Rehab Alnemr, et al.

- Social aspects and usability: such as "The Evolution of Trust" by Pam Briggs, "Why We Need More Effective Trust Signaling" by Angela Sasse, and "Identifying Knots of Trust in Virtual Communities" by Nurit Gal-Oz, et al.

- Trust in the Cloud: such as "Enhancing Data Privacy in the Cloud" by Gene Tsudik et al., and "Regulatory Impact of Data Protection and Privacy in the Cloud" by Srijith K. Nair et al.

In 2012 the IFIP working group of Trust Management took yet another risk. This has been the first time for the working group to organize an event in India. The main motive for hosting an IFIPTM conference in India has been to introduce the trust management discipline to the research, government and commercial innovation communities of the Indian subcontinent and engage them into the research fostered by IFIP on Trust Management. Part of the motivation has also been to illustrate the catalyst role that Trust Management methods, techniques and know-how can play in a rapidly emerging economy and to a society that has yet another significantly different fabric and foundation than those of Europe, North America and Japan. The impact of IFIPTM 2012 in India is yet to be experienced and analyzed but the first indications from the IFIPTM Winter School in Surat in early 2012 are encouraging and show a high level of interest and likely involvement from the local research communities.

\section{Concluding Remarks}

The mission of the IFIP Trust Management is even more relevant now than the beginning of the $21^{\text {st }}$ century. Trust remains a fundamental consideration for the growth and stability of electronic markets and on-line communities. Trust guides decisions about on-line interactions between humans, decisions about which service to consume and how and decisions about how organizations conduct their business and how they engage in business partnerships. The emergence of Cloud computing the establishment of interconnected social networks, covering now most social activities in modern life, and the proliferation of personal devices, electronic media and smart appliances offering continuous connectivity to on-line services from mixed home and work environments, bring about a situation that necessitates a radical rethinking of old security and on-line interaction models to meet new challenges. These challenges are different than those of 2001 but still highlight how relevant and important it is to understand and manage trust and to make trust-based decisions. Nurturing this know-how is critical not only for improving our on-line experience, but also for avoiding another drop in consumer or corporate confidence in the new technologies and new ways of social and business conduct, of higher magnitude than the dot-com bubble burst of 2001 . 
Failure to assess trust in online environments may lead to multiple and diverse security problems: The exploitation of global network mechanisms can enable attackers to disrupt services on a massive scale. Individuals or organized groups of criminals may also use automated agents to exploit market platforms to commit fraud and gain unfair advantages. Cleverly designed deceptions can trick a significant percentage of online users into revealing sensitive information. Online media and communities can be manipulated to create unnatural opinion biases and to hijack democratic processes. There is still relatively little technology support available for assessing the reliability and good faith of entities and the quality of resources in online environment, while people have a higher tendency to deceive through online interactions (compared to interaction involving physical presence). This increases uncertainty and risk, and it is in this environment that online communities and markets grow rapidly these days.

Contemporary research in trust management has two main facets, aligned with the nature of trust relationships. Firstly, it is about the relying parties assessing the reliability and good faith of other parties, as well of assessing the security, reliability and quality of online services, and helping to make better decisions about which parties it is safe to transact with. Secondly, it is about designing reliable and secure systems and processes, and enabling participants in online markets and communities to establish themselves as worth being trusted. Contemporary research in trust management enables providing incentives for good faith and quality services and sanctioning lowquality services and deceptive behavior has the effect of stimulating the emergence of quality markets and communities. It continues to bring together methods and tools from multiple disciplines including policy, information and network security, artificial intelligence, law, and cognitive sciences.

Ten years after the dot com burst in the "developed" economies of northern Europe, North America, and the Far East, there are similarities between the challenges that these economies faced while rebuilding trust in their on-line services and their on-line communities and the challenges that the emerging mega-economies, such as this in the Indian subcontinent, and elsewhere in Asia, in the Middle East, in South Africa and in Latin America, face now and will continue to face in the near future.

I think that academics, professionals, and entrepreneurs in today's emerging megaeconomies can benefit by understanding trust and trust management and by studying the achievements and pitfalls of the "old-world" ageing economies that had to rebuild trust in on-line communities and on-line services, once or more, over the last ten years. It is my hope and expectation that the IFIP working group on Trust Management will play a catalyst role in this evolution and help to pave a way for a free, robust and resilient on-line market as the dynamics of the global economy evolve and new opportunities appear and growth shifts from the mature and declining economies of the West to the immature but vibrant and rapidly growing economies of the East.

I wish that the IFIPTM 2012 event in Surat India plays a very fruitful and pioneering role in this direction. 


\section{References}

1. Gambetta, D.: Can We Trust Trust? In: Gambetta, D. (ed.) Trust: Making and Breaking Cooperative Relations, electronic edn., Department of Sociology, pp. 213-237. University of Oxford (1998),

http: / / www.sociology.ox.ac.uk/papers/gambetta213-237.pdf

2. McKnight, D.H., Chervany, N.L.: The Meanings of Trust. Technical Report MISRC Working Paper Series 96-04, University of Minnesota, Management Information Systems Research Center (1996), http: / / misrc.umn. edu/wpaper/

3. March, S.P.: Formalising Trust as a Computational Concept. In: Computing Science and Mathematics, p. 170. University of Stirling (1994)

4. Jøsang, A.: Artificial Reasoning with Subjective Logic. In: 2nd Australian Workshop on Commonsense Reasoning (1997),

http: / / www.idt.ntnu.no/ ajos/papers.html

5. Jøsang, A.: A Subjective Metric of Authentication. In: Quisquater, J.-J., Deswarte, Y., Meadows, C., Gollmann, D. (eds.) ESORICS 1998. LNCS, vol. 1485, pp. 329-344. Springer, Heidelberg (1998), http: / / www.idt.ntnu.no/ ajos/papers.html

6. Jøsang, A.: The right type of trust for distributed systems. In: ACM New Security Paradigms Workshop (1996), http: / / www.idt.ntnu.no/ ajos/papers.html

7. Jøsang, A.: A Logic for Uncertain Probabilities. International Journal of Uncertainty, Fuzziness and Knowledge-Based Systems 9(3), 279-311 (2001)

8. Blaze, M., Feigenbaum, J., Lacy, J.: Decentralized Trust Management. In: IEEE Conference on Security and Privacy, Oakland, California, USA (1996),

http: / /www. crypto.com/papers/policymaker.pdf

9. Blaze, M., Ioannidis, J., Keromytis, A.D.: Experience with the KeyNote Trust Management System: Applications and Future Directions. In: Nixon, P., Terzis, S. (eds.) iTrust 2003. LNCS, vol. 2692, pp. 284-300. Springer, Heidelberg (2003)

10. Anderson, S., et al.: Web Services Trust Language (WS-Trust) (2005), http: / / specs. xmlsoap.org/ws/2005/02/trust/WS-Trust.pdf

11. Grandison, T., Sloman, M.: A survey of trust in Internet applications. IEEE Communications Surveys and Tutorials 3, 2-16 (2000)

12. Ruohomaa, S., Kutvonen, L.: Trust Management Survey. In: Herrmann, P., Issarny, V., Shiu, S.C.K. (eds.) iTrust 2005. LNCS, vol. 3477, pp. 77-92. Springer, Heidelberg (2005)

13. Jøsang, A., Ismail, R., Boyd, C.: A Survey of Trust and Reputation Systems for Online Service Provision. Decision Support Systems 43(2) (2007)

14. Jøsang, A., Keser, C., Dimitrakos, T.: Can We Manage Trust? In: Herrmann, P., Issarny, V., Shiu, S.C.K. (eds.) iTrust 2005. LNCS, vol. 3477, pp. 93-107. Springer, Heidelberg (2005)

15. Willoughby, J.: "Burning Up", article in Barrons magazine, March 20 (2000)

16. Dimitrakos, T.: System Models, e-Risks and e-Trust. In: I3E 2001, pp. 45-58 (2001)

17. Dimitrakos, T.: A Service-Oriented Trust Management Framework. In: Falcone, R., Barber, S.K., Korba, L., Singh, M.P. (eds.) AAMAS 2002 Ws Trust, Reputation... LNCS (LNAI), vol. 2631, pp. 53-72. Springer, Heidelberg (2003)

18. Grandison, T., Sloman, M.: Specifying and Analysing Trust for Internet Applications. In: Proceedings of the 2nd IFIP Conference on e-Commerce, e-Business and e-Government (I3E 2002), Lisbon (2002)

19. Maibaum, T.S.E.: How Do I Trust Thee? Let Me Count The Ways. In: IEEE ICCI 2002, p. 23 (2002) 
20. Jones, A.J.I., Firozabadi, B.: On the characterisation of a trusting agent - aspects of a formal approach. In: Castelfranchi, C., et al. (eds.) Trust and Deception in Virtual Societies, pp. 157-168. Kluwer Academic Publishers, Dordrecht (2001)

21. Falcone, R., Castelfranchi, C.: The Socio-Cognitive Dynamics of Trust: Does Trust Create Trust? In: Falcone, R., Singh, M., Tan, Y.-H. (eds.) Trust in Cyber-societies. LNCS (LNAI), vol. 2246, pp. 55-72. Springer, Heidelberg (2001)

22. Castelfranchi, C., Falcone, R.: Trust is Much More Than Subjective Probability: Mental Components and Sources of Trust. In: HICSS 2000 (2000)

23. Jones, S., Wilikens, M., Morris, P., Masera, M.: Trust Requirements in E-Business: A Conceptual Framework. Communications of the ACM 43 (December 2000)

24. Dimitrakos, T., Raptis, D., Ritchie, B., Stølen, K.: Model Based Security Risk Analysis for Web Applications. In: EuroWeb 2002, Workshops in Computing BCS 2002 (2002)

25. Fredriksen, R., Kristiansen, M., Gran, B.A., Stølen, K., Opperud, T.A., Dimitrakos, T.: The CORAS Framework for a Model-Based Risk Management Process. In: Anderson, S., Bologna, S., Felici, M. (eds.) SAFECOMP 2002. LNCS, vol. 2434, pp. 94-105. Springer, Heidelberg (2002)

26. Nixon, P., Terzis, S. (eds.): iTrust 2003. LNCS, vol. 2692. Springer, Heidelberg (2003)

27. Jensen, C., Poslad, S., Dimitrakos, T. (eds.): iTrust 2004. LNCS, vol. 2995. Springer, Heidelberg (2004)

28. Dimitrakos, T., Golby, D., Kearney, P.: Towards a Trust and Contract Management Framework for Dynamic Virtual Organisations. In: eAdoption and the Knowledge Economy: eChallenges 2004 (2004)

29. Herrmann, P., Issarny, V., Shiu, S. (eds.): iTrust 2005. LNCS, vol. 3477. Springer, Heidelberg (2005)

30. Stølen, K., Winsborough, W.H., Martinelli, F., Massacci, F. (eds.): iTrust 2006. LNCS, vol. 3986. Springer, Heidelberg (2006)

31. Dimitrakos, T., Martinelli, F.: Formal Aspects in Security and Trust: Second IFIP TC1 WG1.7 Workshop on Formal Aspects in Security and Trust (FAST), an event of the 18th IFIP World Computer Congress, Toulouse, France, August 22-27, 2004. Springer (2005)

32. Dimitrakos, T., Martinelli, F., Ryan, P.Y.A., Schneider, S. (eds.): FAST 2005. LNCS, vol. 3866. Springer, Heidelberg (2006)

33. Dimitrakos, T., Martinelli, F., Ryan, P.Y.A., Schneider, S. (eds.): FAST 2006. LNCS, vol. 4691. Springer, Heidelberg (2007)

34. Degano, P., Guttman, J., Martinelli, F. (eds.): FAST 2008. LNCS, vol. 5491. Springer, Heidelberg (2009)

35. Etalle, S., Marsh, S. (eds.): Trust Management. IFIP, vol. 238. Springer, Boston (2007)

36. Karabulut, Y., Mitchell, J., Herrmann, P., Jensen, C.D. (eds.): Trust Management II. IFIP, vol. 263. Springer, Boston (2008)

37. Ferrari, E., Li, N., Bertino, E., Karabulut, Y. (eds.): IFIPTM 2009. IFIP AICT, vol. 300. Springer, Heidelberg (2009)

38. Nishigaki, M., Jøsang, A., Murayama, Y., Marsh, S. (eds.): IFIPTM 2010. IFIP AICT, vol. 321. Springer, Heidelberg (2010)

39. Wakeman, I., Gudes, E., Jensen, C.D., Crampton, J. (eds.): IFIPTM 2011. IFIP AICT, vol. 358. Springer, Heidelberg (2011) 